\title{
An assessment of mumps vaccine effectiveness by dose during an outbreak in Canada
}

\author{
Shelley L. Deeks MD MHSc, Gillian H. Lim MSc, Mary Anne Simpson MSc, Louise Gagné MSc, \\ Jonathan Gubbay MBBS MMedSc, Erik Kristjanson BSc MLT, Cecilia Fung MPH, Natasha S. Crowcroft MD PhD
}

Competing interests: Jonathan Gubbay received a research grant from GlaxoSmithKline and HoffmanLa Roche to study antiviral resistance in influenza. No other competing interests were declared.

This article has been peer reviewed.

Correspondence to: Dr. Shelley L. Deeks, shelley.deeks@oahpp.ca

CMAJ 2011. DOI:10.1503 /cmaj.101371

\section{ABSTRACT}

Background: This investigation was done to assess vaccine effectiveness of one and two doses of the measles, mumps and rubella (MMR) vaccine during an outbreak of mumps in Ontario. The level of coverage required to reach herd immunity and interrupt community transmission of mumps was also estimated.

Methods: Information on confirmed cases of mumps was retrieved from Ontario's integrated Public Health Information System. Cases that occurred between Sept. 1, 2009, and June 10, 2010, were included. Selected health units supplied coverage data from the Ontario Immunization Record Information System. Vaccine effectiveness by dose was calculated using the screening method. The basic reproductive number $\left(R_{0}\right)$ represents the average number of new infections per case in a fully susceptile population, and $R_{0}$ values of between 4 and 10 were considered for varying levels of vaccine effectiveness.
Results: A total of 134 confirmed cases of mumps were identified. Information on receipt of MMR vaccine was available for $114(85.1 \%)$ cases, of whom $63(55.3 \%)$ reported having received only one dose of vaccine; $32(28.1 \%)$ reported having received two doses. Vaccine effectiveness of one dose of the MMR vaccine ranged from $49.2 \%$ to $81.6 \%$, whereas vaccine effectiveness of two doses ranged from $66.3 \%$ to $88.0 \%$. If we assume vaccine effectiveness of $85 \%$ for two doses of the vaccine, vaccine coverage of $88.2 \%$ and $98.0 \%$ would be needed to interrupt community transmission of mumps if the corresponding reproductive values were four and six.

Interpretation: Our estimates of vaccine effectiveness of one and two doses of mumpscontaining vaccine were consistent with the estimates that have been reported in other outbreaks. Outbreaks occurring in Ontario and elsewhere serve as a warning against complacency over vaccination programs.
$\mathrm{B}$ etween September 2009 and June 2010, there was an outbreak of mumps in Ontario, Canada. Outbreaks of mumps were also taking place in the United States (New York and New Jersey) and in Israel during the same period. ${ }^{12}$ These outbreaks shared several features, including the age distribution of the cases, the predominance of male cases and the occurrence of the disease among people who had received vaccinations against mumps. ${ }^{12}$ This latter issue can lead to questions from the public and health care providers regarding the effectiveness of the vaccine. Rapid assessment of vaccine effectiveness is, therefore, an important component of outbreak management for vaccine-preventable diseases, and knowledge of the history of the vaccination program is necessary for interpreting the results.

A live attenuated mumps vaccine was licensed in Canada in 1969 and introduced in Ontario shortly thereafter. In 1975, a single dose of the combined vaccine for measles, mumps and rubella (MMR) was implemented. A single-dose program continued until 1996, when a second dose of the MMR vaccine was introduced as part of a plan to eradicate measles. The first dose of the MMR vaccine is routinely given at 12 months of age; until 2007, the second dose was recommended for children between four and six years of age. The recommended age for the second dose is now 18 months.

Two MMR vaccines are available for use in Ontario. A single dose of monovalent measles vaccine was offered to all students aged 4-18 years in 1996 as part of a measles catch-up campaign. Because mumps-containing vaccine was not used, a cohort of individuals born before 1992 who had only received one dose of mumps-containing vaccine were therefore potentially susceptible to the virus.

Epidemiologic data show that the mumps virus circulated relatively widely throughout Ontario until approximately 1980 (M.A. Simpson, Ontario Ministry of Health and Long-Term Care, Toronto, Ont.: personal communication, 2011 Feb. 1), which would have resulted in the 
natural boosting of the population's immunity to the disease. The combination of changes in the policy governing vaccination against mumps and the circulation of the disease thus resulted in a susceptible cohort of individuals born between approximately 1980 and 1992 (i.e., people currently between 19 and 31 years of age).

A person's susceptibility to mumps is also influenced by the effectiveness of the vaccine he or she received. Clinical trials have reported vaccine efficacy of approximately $95 \%$ after one dose of mumps vaccine under controlled conditions, although estimates of vaccine effectiveness that were conducted in the field (as opposed to clinical trials) have been much lower (i.e., $62 \%-85 \%)$. $^{3}$ There is less information available about vaccine effectiveness after two doses, but estimates have ranged from $76 \%$ to $95 \%,{ }^{3-5}$ with accumulating evidence of waning immunity..$^{2-6}$

The objectives of this investigation were to assess the vaccine effectiveness of MMR vaccine by dose and by birth cohort during the outbreak, and to estimate the level of vaccine coverage required to reach herd immunity and interrupt community transmission of mumps.

\section{Methods}

The outbreak period was defined as Sept. 1, 2009, to June 10, 2010. Vaccination data on cases occurring during this period were provided by all Ontario health units with confirmed cases of mumps. The six health units with the highest incidence of mumps supplied data on vaccine coverage by birth cohort from the Ontario Immunization Record Information System (IRIS) database. Coverage was assessed as of Apr. 30, 2009, as this followed a provincial mumps vaccine catch-up campaign that targeted students at postsecondary institutions. Information on each case was retrieved from the integrated Public Health Information System (iPHIS) as of Aug. 9, 2010. Additional data on immunization was requested from health units as needed. Two cases were reclassified as having received one dose of MMR vaccine instead of two because their symptoms started within eight weeks of receiving the second dose.

Any blood samples provided by patients for laboratory testing were submitted to the Ontario Agency for Health Protection and Promotion for diagnostic serology. The specimens were tested for seroconversion of mumps immunoglobulin $\mathrm{G}$ and immunoglobulin $\mathrm{M}$ using commercially available enzyme-linked immunoassay kits (Euroimmun, Lübeck, Germany and Siemens, Marburg, Germany). Respiratory tract, urine and cerebrospinal fluid samples were tested by viral culture and inhouse real-time reverse-transcriptase polymerase chain reaction adapted from previously published methods. ${ }^{7,8}$ Confirmed cases were designated as confirmed in iPHIS or cases satisfying the outbreak case definition (Box 1). ${ }^{9}$

Case and coverage data by dose were used to calculate vaccine effectiveness using the screening method (Box 2).$^{10}$ Only people born between 1980 and 2002 whose vaccination status was known were included in this analysis because of the distribution of the cases' ages and issues with the quality of the IRIS data for the oldest cohort. The year 2002 was selected, as this is when children would have been considered overdue for their second dose

\section{Box 1: Criteria for the definition of a confirmed case of mumps during an outbreak in Ontario}

A confirmed case includes anyone* with symptom onset between Sept. 1, 2009, and June 10,2010, with any of the following in the absence of recent immunization with mumps-containing vaccine (i.e., within the last 28 days)

- Isolation of mumps virus from an appropriate clinical specimen (e.g., buccal swab or urine sample) in a person with clinically compatible illnesst, or fever or any respiratory symptoms

- Detection of mumps virus ribonucleic acid (RNA) using a validated nucleic acid amplification test (NAT) from an appropriate clinical specimen (e.g., buccal swab or urine sample) in a person with clinically compatible illnesst, or fever or any respiratory symptoms

- Demonstration of seroconversion or a significant, as determined by the laboratory, rise in mumps immunoglobulin G (IgG) antibody level between the acute and convalescent sera in a person with clinically compatible illnesst, or fever or any respiratory symptoms

- Positive serologic test for mumps immunoglobulin M (IgM) antibody using a recommended assay in an individual with clinically compatible illness $\dagger$

- Clinically compatible illnesst in a person with an epidemiologic link

*Persons who travelled to a mumps-endemic country within 25 days of their illness onset were excluded.

tClinically compatible illness consists of acute onset of unilateral or bilateral tenderness and/or self-limited swelling of the parotid gland or other salivary gland, lasting $>2$ days, and without other apparent cause.

$\ddagger$ An epidemiologic link to either a laboratory-confirmed case or to a cluster of cases in which at least one case is laboratory confirmed.

Source: Ontario mumps epidemiologic summary - Appendix I: Ontario mumps case definition for outbreak number 0000-2009-011. Ontario Ministry of Health and Long-Term Care, Toronto, Ont. ${ }^{9}$

\section{Box 2: Formula to calculate vaccine effectiveness by dose,} using the screening method $^{10}$

$$
\mathrm{VE}_{i}=1-\left(\frac{\mathrm{PCV}_{i}}{1-\mathrm{PCV}_{i}} \times \frac{1-\mathrm{PPV}_{i}}{\mathrm{PPV}_{i}}\right)
$$

where $\mathrm{VE}_{i}$ is the vaccine effectiveness of $i$ doses, $\mathrm{PCV}_{i}$ is the proportion of cases vaccinated with $i$ doses, $\mathrm{PPV}_{i}$ is the proportion of the population vaccinated with $i$ doses, and $i$ is 1 or 2 .

$\mathrm{PCV}_{i}$ can be calculated as follows:

$$
\mathrm{PCV}_{i}=\frac{\text { No. of cases who received } i \text { doses }}{\begin{array}{l}
\text { No. of cases who } \\
\text { received } i \text { doses }
\end{array}+\begin{array}{l}
\text { No. of cases who } \\
\text { received } 0 \text { doses }
\end{array}}
$$

$\mathrm{PPV}_{i}$ can be calculated as follows:

$$
\mathrm{PPV}_{i}=\frac{\text { No. of people in population who received } i \text { doses }}{\begin{array}{c}
\text { No. of people in population } \\
\text { who received } i \text { doses }
\end{array}+\begin{array}{c}
\text { No. of people in population } \\
\text { who received } 0 \text { doses }
\end{array}}
$$


of MMR vaccine when coverage was assessed (i.e., April 2009). The cohorts were selected based on considerations of programmatic issues (allowing for program implementation), coverage, waning immunity and the distribution of cases.

When estimating the effectiveness of one dose, people who had received two doses were excluded from the calculations of the proportions of cases and the population vaccinated. Similarly, people who had received one dose were excluded from calculations that estimated the effectiveness of two doses. Only people born after 1990 were eligible for two doses, so the vaccine effectiveness of two doses was only calculated for birth cohorts between 1990 and 2002. Finally, vaccine effectiveness can only be calculated if at least one of the cases in the cohort of interest had not received a vaccination. Ninety-five per cent confidence intervals were derived using the exact method based on the binomial distribution owing to small numbers. Sensitivity analyses were done to explore the effects of underestimating receipt of the mumps vaccine among cases (scenario 1), as well as both underand overreporting vaccine coverage in the population (scenario 2). For scenario 1, we assumed that all patients whose vaccination status was unknown received zero, one and two doses of MMR vaccine with equal probability; for scenario 2 , we assumed that the proportion of the population vaccinated either decreased or increased by both $3 \%$ and $5 \%$.

Basic reproductive values between 4 and 10 were considered for varying levels of vaccine effectiveness to estimate the level of coverage required to reach herd immunity and interrupt community transmission of the virus (i.e., the herd immunity threshold). This range was used because it is commonly cited in the literature..$^{11,12}$ The basic reproductive number $\left(R_{0}\right)$ represents the average number of new infections per case in a fully susceptible population. The level of immunity in the population required to stop transmission $(I)$ was calculated using the following formula: $I=\left(1-1 / R_{0}\right) \times 100 \%$. The level of coverage required to reach herd immunity $(C)$ for various levels of vaccine effectiveness could not exceed $100 \%$ and was calculated as $C=I / \mathrm{VE}$, where $\mathrm{VE}$ is vaccine effectiveness.

\section{Results}

A total of 134 confirmed cases of mumps were identified in Ontario during the outbreak period. Of these cases, $72.3 \%(n=97)$ had been confirmed with laboratory tests. Figure 1 shows the epidemic curve by the number of doses of MMR vaccine that each case received. The mean age of cases was 25.9 years (median 21 years, range $6-70$ years), $72.4 \%$ of cases were male $(n=97)$, and $58.6 \%$ ( $n=$ 78) of cases were between 15 and 24 years of age.

Information on receipt of mumps vaccine was available for $114(85.1 \%)$ cases; of these, 19 (14.2\%) had received no vaccination, $63(55.3 \%)$ had received one dose of vaccine and 32 (28.1\%) had received two doses of vaccine. Table 1 shows the distribution of mumps cases and the Ontario population by birth cohort; the age distribution of cases was significantly different from the distribution seen in the general population $\left(\chi^{2}\right.$ test, $p<$

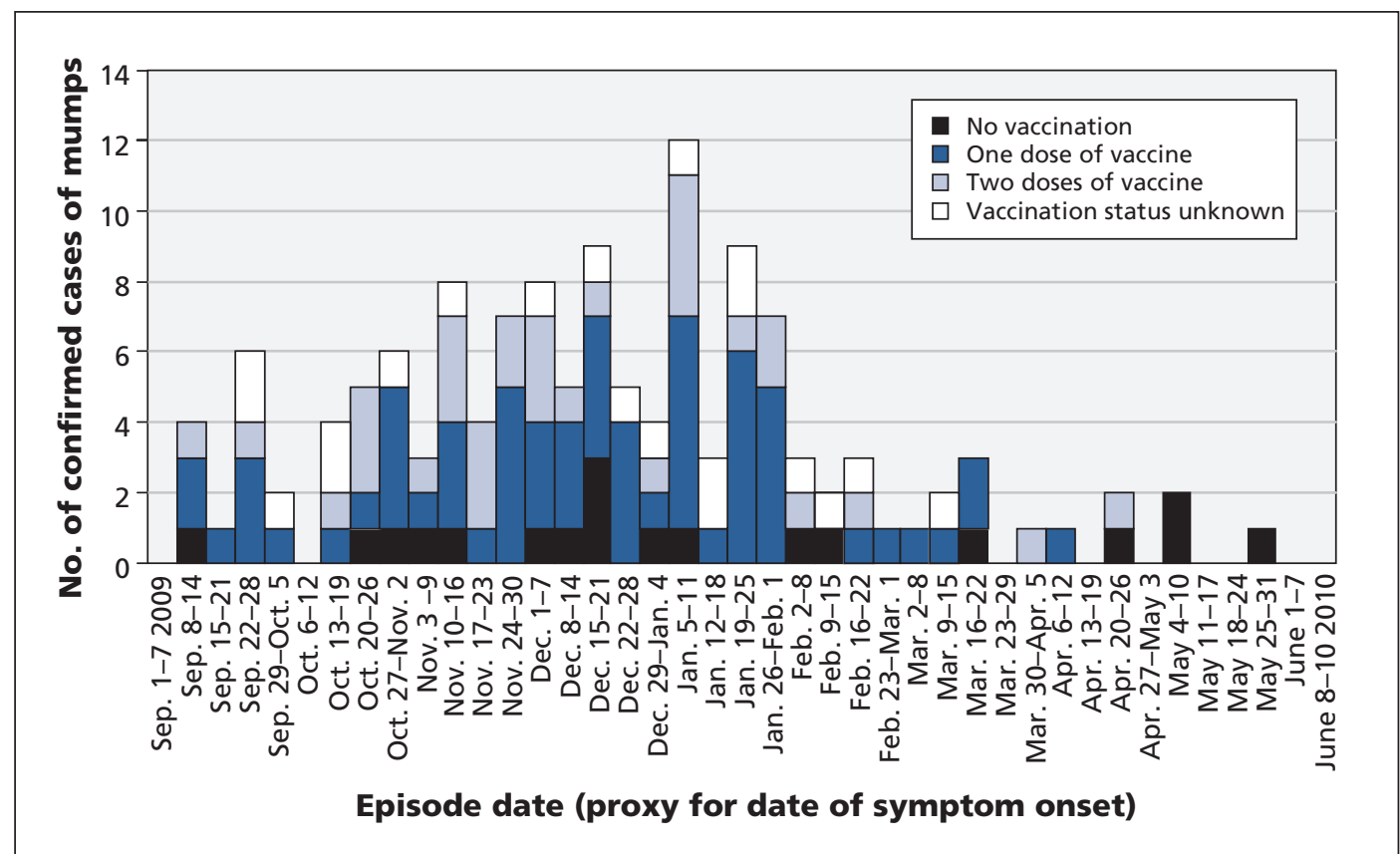

Figure 1: Distribution of confirmed cases of mumps in Ontario by episode date between Sept. 1, 2009, and June 10, $2010(n=134)$. 
0.001). People born between 1980 and 1994 were more likely than expected to be cases based on the distribution of the population (i.e., ratio > 1). This tendency was especially noteworthy among people born between 1985 and 1991 (ratio 4.2-5.1).

MMR vaccination coverage by birth cohort and number of vaccine doses is provided in Figure 2; the estimated vaccine effectiveness of the different doses by birth cohort is presented in Table 2. For our analysis, 88 (65.7\%) cases born between 1980 and 2002 with known vaccine status were included in the assessment of vaccine effectiveness. Vaccine effectiveness of one dose ranged from $49.2 \%$ to $81.6 \%$, whereas the effectiveness of two doses ranged from $66.3 \%$ to $88.0 \%$. The sensitivity analyses showed that if all cases for whom vaccine status was unknown were assumed to have received zero, one or two doses of the MMR vaccine with equal probability, then the vaccine effectiveness for the affected birth cohorts would increase, particularly for people born between 1992 and 1994 (Table 3). If the vaccine coverage was either under- or overestimated (i.e., scenario 2), the corresponding effectiveness would also be under- or overestimated.

The coverage required to reach herd immunity for varying reproductive numbers and different estimates of vaccine effectiveness is provided in Table 4. As the reproductive number increases, so does the level of immunity required in the population to stop transmission of the disease. For example, for a basic reproductive value of four, the corresponding proportion of the population that would need to be immune to mumps to stop the disease from spreading would be $75 \%$; if the reproductive value is $10,90 \%$ of the population would need to be immune to the disease to prevent community transmission. As vaccine effectiveness decreases, higher coverage is required to reach the herd immunity threshold for a given reproductive number. If we assume a two-dose vaccine effectiveness of $85 \%$, we would require $88 \%$ mumps vaccine coverage to interrupt community transmission of the disease for a corresponding reproductive number of four.

\section{Interpretation}

Most of the cases of mumps in this outbreak were male, were 15-24 years of age and were not fully vaccinated. The clustering of cases, particularly among people born between 1985 and 1991, reflects the susceptible cohort, as described previously. In addition, the active social lifestyle of this age group may have facilitated the transmission of the disease. The predominance of male cases was likely due to the settings in which they were exposed to the virus. These settings included ath- letic events, such as hockey tournaments, where there is frequent close contact between people.

The vaccine effectiveness of two doses of MMR vaccine was consistently higher than the

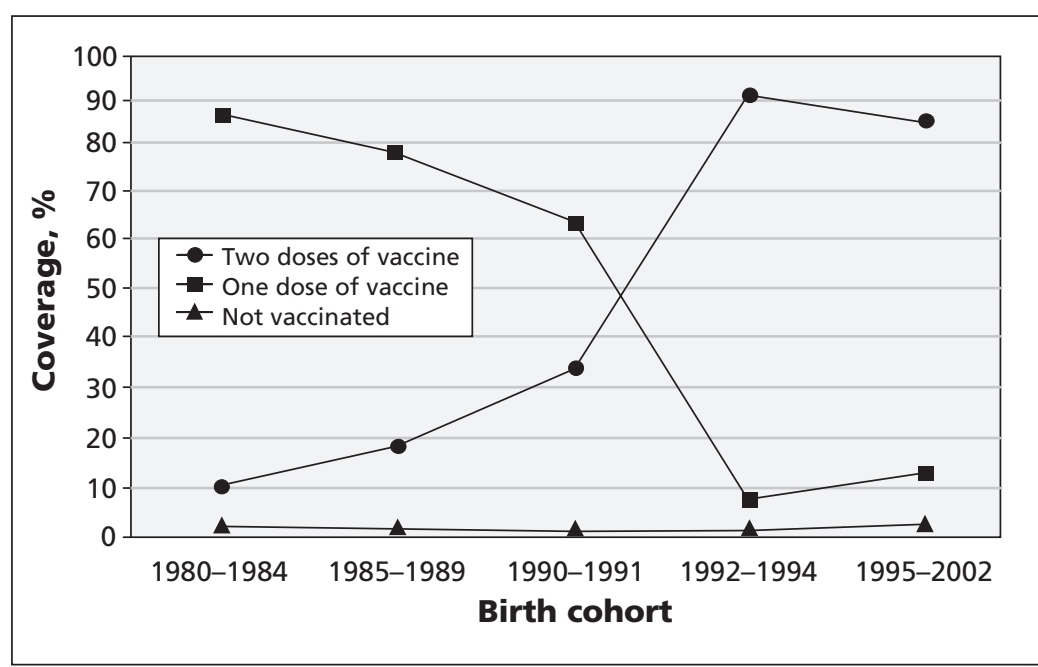

Figure 2: Coverage of measles, mumps and rubella vaccine by number of doses and birth cohort. (Doses were given as of Apr. 30, 2009, according to data in the Immunization Registry Information System from six Ontario health units.)

\begin{tabular}{|c|c|c|c|c|c|}
\hline \multirow[b]{2}{*}{ Birth cohort } & \multicolumn{2}{|c|}{ Population in 2008} & \multicolumn{2}{|c|}{ Confirmed cases of mumps } & \multirow[b]{2}{*}{ Ratiof } \\
\hline & No. & $\%$ of total & No. & $\%$ of total & \\
\hline$<1980$ & 8230430 & 63.7 & 36 & 27.1 & 0.4 \\
\hline 1980-1984 & 876561 & 6.8 & 11 & 8.3 & 1.2 \\
\hline 1985-1989 & 897029 & 6.9 & 39 & 29.3 & 4.2 \\
\hline $1990-1991$ & 361380 & 2.8 & 19 & 14.3 & 5.1 \\
\hline 1992-1994 & 511838 & 4.0 & 20 & 15.0 & 3.8 \\
\hline 1995-2002 & 1212031 & 9.4 & 7 & 5.3 & 0.6 \\
\hline$>2002$ & 839727 & 6.5 & 1 & 0.8 & 0.1 \\
\hline Total & 12928996 & 100.0 & 133 & 100.0 & 1.0 \\
\hline
\end{tabular}

*One case was excluded because the date of birth was unknown.

tProportion of total number of cases divided by proportion of total population.

Table 2: Estimates of vaccine effectiveness for one and two doses of the MMR vaccine by birth cohort $(n=88)$

\begin{tabular}{|lccccccc}
\hline & \multicolumn{3}{c}{ No. of cases } & & \multicolumn{2}{c}{$\begin{array}{c}\text { Vaccine effectiveness, } \\
\%(95 \% \mathrm{Cl})\end{array}$} \\
\cline { 2 - 4 } \cline { 7 - 8 } $\begin{array}{l}\text { Birth } \\
\text { cohort }\end{array}$ & $\begin{array}{c}\text { No } \\
\text { vaccine }\end{array}$ & $\begin{array}{c}\text { One } \\
\text { dose }\end{array}$ & $\begin{array}{c}\text { Two } \\
\text { doses }\end{array}$ & & One dose & Two doses \\
\hline $1980-1984$ & 2 & 6 & 2 & & $81.6(0-96.4)$ & NA* $^{*}$ \\
\hline $1985-1989$ & 3 & 29 & 4 & & $59.4(0-86.2)$ & NA* $^{*}$ \\
\hline $1990-1991$ & 2 & 11 & 3 & & $76.7(0-94.6)$ & $88.0(0-98.6)$ \\
\hline $1992-1994$ & 1 & 2 & 16 & & $49.2(0-97.4)$ & $66.3(0-94.7)$ \\
\hline $1995-2002$ & 1 & 1 & 5 & $76.5(0-99.7)$ & $83.9(0-98.2)$ \\
\hline
\end{tabular}

Note: $\mathrm{Cl}=$ confidence interval, $\mathrm{MMR}=$ measles-mumps-rubella, $\mathrm{NA}=$ not available.

*Data not shown. Analyses were limited to people who were eligible to receive two doses of MMR vaccine through the routine immunization program. 
effectiveness of one dose for each of the cohorts. The results of the sensitivity analyses show how important it is to accurately classify cases according to their vaccination status and to obtain accurate data on vaccine coverage within a population. Immunization status was unknown for 20 cases (14.9\%) in this study. If cases for whom vaccination status was unknown were more likely not to have been vaccinated, then the estimates of vaccine effectiveness would be higher. For example, the vaccine effectiveness for two doses among people born between 1990 and 1991 would have increased from $88 \%$ to $95 \%$ if all cases with unknown vaccine status were unvaccinated (data not shown).

Knowledge of vaccine effectiveness can be used to estimate the level of coverage required to reach herd immunity thresholds. However, the basic reproductive number for mumps is not known with certainty because there are no longer populations fully susceptible to the disease. We therefore used a range of reproductive numbers to assess herd immunity. ${ }^{11,12}$ The two-dose coverage seen in the older cohorts in this investigation was far below the coverage needed to stop transmission of the disease. Targets for coverage set at the national level recommend that $97 \%$ of children should be vaccinated by their seventh birthday. ${ }^{13}$ However, achieving this target would result in herd immunity under only some of the circumstances outlined in Table 4 (e.g., a reproductive number of four and a vaccine effectiveness of $80 \%$ or higher).

Mumps outbreaks have become relatively unusual in Canada and in other countries that have implemented two-dose MMR vaccination programs. In the past, it was not uncommon to have outbreaks among people who had received only one dose of a mumps-containing vaccine. However, that had not been the case since the implementation of the two-dose policies. In fact, outbreaks occurring predominantly among recipients of two doses of vaccine were rarely documented anywhere in the world until 2006. ${ }^{14}$ In the 2004 2005 outbreak in the United Kingdom that affected more than 5000 people, approximately two-thirds had not been vaccinated against mumps. ${ }^{15}$ Similarly, in the 2007 outbreaks of mumps in Canada, only $7.7 \%$ (45/586) of the cases whose immunization status was known had received two doses of vaccine. ${ }^{16}$ In contrast, between $62.5 \%$ and $75.2 \%$ of cases reported in more recent outbreaks in Canada,

\begin{tabular}{|c|c|c|c|c|c|c|c|c|}
\hline \multirow[b]{3}{*}{ Coverage } & \multicolumn{8}{|c|}{ Birth cohort; vaccine effectiveness, $\%$} \\
\hline & \multirow{2}{*}{$\begin{array}{c}\text { 1980-1984 } \\
\text { One dose }\end{array}$} & \multirow{2}{*}{$\begin{array}{r}1985-1989 \\
\text { One dose }\end{array}$} & \multicolumn{2}{|c|}{ 1990-1991 } & \multicolumn{2}{|c|}{ 1992-1994 } & \multicolumn{2}{|c|}{ 1995-2002 } \\
\hline & & & One dose & Two doses & One dose & Two doses & One dose & Two doses \\
\hline Baseline & 81.6 & 59.4 & 76.7 & 88.0 & 49.2 & 66.3 & 76.5 & 83.9 \\
\hline Scenario $1 *$ & 87.7 & 73.9 & 83.1 & 89.4 & 74.6 & 83.1 & 76.5 & 83.9 \\
\hline \multicolumn{9}{|l|}{ Scenario $2 *$} \\
\hline PPV $-5 \%$ & 81.9 & 58.7 & 71.0 & 84.7 & $-72.5 t$ & 44.6 & 54.9 & 76.6 \\
\hline PPV $-3 \%$ & 84.3 & 63.2 & 73.4 & 86.2 & 10.4 & 53.6 & 66.7 & 79.6 \\
\hline PPV $+3 \%$ & 90.9 & 75.3 & 79.7 & 89.6 & 65.2 & 78.1 & 82.1 & 87.8 \\
\hline PPV $+5 \%$ & 92.9 & 78.9 & 81.6 & 90.5 & 71.6 & 85.6 & 84.7 & 100.0 \\
\hline
\end{tabular}

Table 4: Estimates of the coverage required to reach herd immunity for varying reproductive numbers and different levels of vaccine effectiveness

\begin{tabular}{|c|c|c|c|c|c|c|}
\hline $\begin{array}{l}\text { Reproductive } \\
\text { number }\end{array}$ & $\begin{array}{l}\text { Level of immunity* } \\
\text { required to stop } \\
\text { transmission, \% }\end{array}$ & \multicolumn{5}{|c|}{ Coverage $t$ required to reach herd immunity for varying levels of vaccine effectiveness, $\%$} \\
\hline 6 & 83.3 & - & - & 98.0 & 92.6 & 87.7 \\
\hline 8 & 87.5 & - & - & - & 97.2 & 92.1 \\
\hline 10 & 90.0 & - & - & - & - & 94.7 \\
\hline
\end{tabular}


the US and Israel had received two doses of mumps-containing vaccine. , $^{1,6,17}$

Inadequate coverage, a susceptible cohort of people who were only eligible to receive one dose of vaccine and waning immunity have been cited as possible explanations for the resurgence of mumps in developed countries..$^{3,4,6,15}$ Cohen and coauthors showed that the effectiveness of two doses of mumps vaccine declined from $98.8 \%$ in children 5-6 years of age to $86.4 \%$ in children 11-12 years of age. ${ }^{3}$ It has been 14 years since Ontario implemented a two-dose policy for MMR vaccine. Therefore we are now at the point in the evolution of the program where we may begin to see the effect of waning immunity among recipients of two doses of the vaccine.

Although the point estimates of vaccine effectiveness seen in this study must be interpreted with caution owing to the small size of the sample, they are generally consistent with the estimates reported in other outbreaks. In a recent review of 50 outbreaks of mumps, the effectiveness of one dose of mumps-containing vaccine was $72.8 \%-91.0 \%$, whereas the effectiveness for two doses was 91.0\%-94.6\%. ${ }^{4}$ Estimates of vaccine effectiveness were slightly lower in the 2006 outbreak in the US, ranging between $64 \%$ and $84 \%$ for one dose, and between $76 \%$ and $88 \%$ for two doses. ${ }^{5}$ Mumps outbreaks that have affected older adolescents and young adults have previously been observed in Canada $^{16-18}$ and in countries where two-dose policies were implemented during the 1990s. ${ }^{6,15,19}$ During the 2007 Canadian outbreak, $58.1 \%$ of cases were between 20 and 29 years of age, ${ }^{16}$ in the 2006 outbreak in the US, the highest attack rates were reported among people aged $18-24$ years. ${ }^{6}$

\section{Limitations}

The estimates of vaccine effectiveness were imprecise owing to the small number of cases in each birth cohort, as reflected by the wide confidence intervals. Although the screening method offers a quick and relatively simple means for assessing vaccine effectiveness, it requires accurate estimates of coverage. Because IRIS is not a comprehensive immunization registry, the coverage data used to derive these estimates have limitations.

Coverage varied among the selected health units; the weighted overall estimate may not have been fully representative of the population from which the cases were derived. Coverage may have been underestimated if immunization was not reported; conversely, coverage may have been overestimated if denominators failed to capture all eligible people.

Finally, we were not able to assess waning immunity due to the small number of cases.

\section{Conclusion}

Accumulating data on vaccine effectiveness and waning immunity may have important implications for immunization policy. All jurisdictions in Canada currently have a two-dose MMR vaccination policy; however, the timing of the second dose varies among the provinces and territories. As of 2009, 10 jurisdictions, including Ontario, offered the second dose at 18 months of age; the remaining three jurisdictions offer the second dose at four to six years of age. ${ }^{20}$ If all jurisdictions administered the second dose of the vaccine to children between the ages of four and six years, this could have an impact on waning immunity. However, it is unlikely that we will be able to eliminate mumps with the current vaccine and vaccination policies and at the current levels of coverage. Improving the coverage of two doses of MMR vaccine is critical to the prevention of further outbreaks of mumps.

Another policy question is whether a second dose of mumps-containing vaccine is needed for the susceptible cohort, notwithstanding the difficulty in accessing this population. Ontario implemented a province-wide mumps vaccination catch-up campaign that targeted students in postsecondary institutions between August 2008 and March 2009, but uptake was poor. ${ }^{21}$

Outbreaks of mumps in Canada and abroad serve as a reminder that we cannot become complacent about vaccination programs or maximizing vaccine coverage. Rapid assessment of vaccine effectiveness can occur as part of the response to an outbreak and is important in maintaining confidence in vaccination programs. Accurate and timely assessment of immunization coverage through the implementation of a comprehensive immunization registry would improve our ability to assess both vaccine effectiveness and waning immunity to the disease. Furthermore, closely monitoring waning immunity will help to ensure that we have the necessary data for making policy decisions, such as whether a third dose of MMR vaccine is necessary or whether a different vaccine should be considered, and for evaluating the cost-effectiveness of the program.

\section{References}

1. Centers for Disease Control and Prevention. Update: mumps outbreak - New York and New Jersey, June 2009-January 2010. MMWR Morb Mortal Wkly Rep 2010;59:125-9. [Erratum in: MMWR Morb Mortal Wkly Rep 2010;59:594. Dosage error in article text.]

2. Stein-Zamir C, Shoob H, Abramson N, et al. Mumps outbreak in Jerusalem affecting mainly male adolescents. Euro Surveill 2009;14:1-3. Available: www.eurosurveillance.org/ViewArticle .aspx?ArticleId=19440 (accessed 2010 Aug. 26.)

3. Cohen C, White JM, Savage EJ, et al. Vaccine effectiveness estimates, 2004-2005 mumps outbreak, England. Emerg Infect Dis 2007; 13:12-7.

4. Dayan GH, Rubin S. Mumps outbreaks in vaccinated populations: Are available mumps vaccines effective enough to prevent outbreaks? Clin Infect Dis 2008;47:1458-67. 
5. Marin M, Quinlisk P, Shimabukuro T, et al. Mumps vaccination coverage and vaccine effectiveness in a large outbreak among college students - Iowa, 2006. Vaccine 2008;26:3601-7.

6. Dayan GH, Quinlisk MP, Parker AA, et al. Recent resurgence of mumps in the United States. N Engl J Med 2008;358:1580-9.

7. Uchida K, Shinohara M, Shimada S, et al. Rapid and sensitive detection of mumps virus RNA directly from clinical samples by real-time PCR. J Med Virol 2005;75:470-4.

8. Boddicker JD, Rota PA, Kreman T, et al. Real-time reverse transcription-PCR assay for detection of mumps virus RNA in clinical specimens. J Clin Microbiol 2007;45:2902-8.

9. Ontario mumps epidemiologic summary - Appendix I: Ontario mumps case definition for outbreak number 0000-2009-011. Toronto $(\mathrm{ON})$ : Ontario Ministry of Health and Long-term Care; 2010.

10. Farrington CP. Estimation of vaccine effectiveness using the screening method. Int J Epidemiol 1993;22:742-6.

11. Fine PE. Herd immunity: history, theory, practice. Epidemiol Rev 1993;15:265-302.

12. Anderson RM, May RM. Immunisation and herd immunity. Lancet 1990;335:641-5.

13. Public Health Agency of Canada. Final report to outcomes from the National Consensus Conference for Vaccine-Preventable Diseases in Canada, June 12-14, 2005 — Québec City, Quebec. Can Commun Dis Rep 2008;34(Suppl 2):1-56.

14. Barskey AE, Glasser JW, LeBaron CW. Mumps resurgences in the United States: a historical perspective on unexpected elements. Vaccine 2009;27:6186-95.

15. Centers for Disease Control and Prevention. Mumps epidemic - United Kingdom, 2004-2005. MMWR Morb Mortal Wkly Rep 2006;55:173-5.

16. Public Health Agency of Canada. Guidelines for the prevention and control of mumps outbreaks in Canada. Can Commun Dis Rep 2010;36S1:1-46.

17. Watson-Creed G, Saunders A, Scott J, et al. Two successive outbreaks of mumps in Nova Scotia among vaccinated adolescents and young adults. CMAJ 2006; 175:483-8.

18. Bell A, Fyfe M, Bigham M, et al. Outbreak of mumps among young adults - Vancouver, British Columbia. Can Commun Dis Rep 1997;23:169-72.

19. Whelan J, van Binnendijk R, Greenland K, et al. Ongoing mumps outbreak in a student population with high vaccination coverage, Netherlands, 2010. Euro Surveill 2010;15:1-4. Available: www.eurosurveillance.org/ViewArticle.aspx?ArticleId $=19554$ (accessed 2010 Aug. 26).

20. Publicly funded immunization programs in Canada - routine schedule for infants and children (including special programs and catch-up programs). Ottawa (ON): Public Health Agency of Canada. Available: www.phac-aspc.gc.ca/im/ptimprog -progimpt/table-1-eng.php (accessed 2010 Aug. 26).

21. Immunization coverage report for school pupils. School years 2004/05 to 2007/08. Toronto (ON): Ontario Ministry of Health and Long-Term Care, Public Health Division; 2009.

Affiliations: From the Ontario Agency for Health Protection and Promotion (Deeks, Lim, Gubbay, Kristjanson, Crowcroft), Toronto, Ont.; the Ministry of Health and Long-Term Care (Simpson, Fung), Toronto, Ont.; the North Bay Regional Health Unit (Gagné), North Bay, Ont.; the Dalla Lana Public School of Health (Deeks, Crowcroft); the Department of Laboratory Medicine and Pathobiology (Gubbay, Crowcroft); and the Department of Paediatrics (Gubbay), University of Toronto, Toronto, Ont.

Contributors: Shelley Deeks designed the investigation and contributed to the validation and analysis of the data, organized and presented the information and interpreted the data; she is the primary author of the article. Gillian Lim compiled, validated and analyzed the data, organized and presented the information, interpreted the data and helped write the article. Mary Anne Simpson contributed to the interpretation of the data. Louise Gagné provided a portion of the primary data, contributed to the interpretation of the data and helped revise the article. Jonathan Gubbay did the laboratory testing that supported the investigation of the outbreak and helped write the article. Erik Kristjanson provided a portion of the primary data and interpreted the laboratory results. Cecilia Fung supported queries surrounding the data extracted from iPHIS. Natasha Crowcroft contributed to the validation and interpretation of the analysis and assisted with organizing and presenting the information. 